\title{
Pseudotumor Associated with Dall-Miles Cable Breakage Following Revision Total Hip Arthroplasty: A Case Report
}

\author{
Se Won Oh ${ }^{1}$ (D), Seung Yun Jee ${ }^{2}$ (D), Seong Oh Park ${ }^{1}$ (D) \\ Departments of ${ }^{1}$ Plastic and Reconstructive Surgery and ${ }^{2}$ Pathology, Hanyang University College of Medicine, Seoul, Korea
}

\begin{abstract}
A pseudotumor is a benign granulomatous or cystic lesion associated with the deposition of metal debris in joint arthroplasties. To the best of our knowledge, this is the first report on a pseudotumor caused by the breakage of the Dall-Miles cable grip system, a multifilament cable used to fix the trochanteric osteotomy in revision total hip arthroplasty. This rare case demonstrates that pseudotumor formation can be caused by various types of orthopedic materials and implant components. When encountering a palpable mass around an orthopedic implant, a pseudotumor should be considered along other differential diagnoses. To treat pseudotumors and also prevent recurrence, its complete resection and sufficient removal of orthopedic materials are necessary.
\end{abstract}

Keywords: Pseudotumor; Granuloma; Metallosis; Dall-Miles cable grip system; Arthroplasty

\section{Introduction}

Pseudotumors are benign granulomatous or cystic lesions that are associated with the deposition of metal debris on periprosthetic soft tissues in joint arthroplasties [1]. They can cause tissue necrosis, osteolysis, and prosthetic dislocation. While clinical manifestations of pseudotumors include discomfort and pain with palpable swelling, and neuropathy or venous compression due to the mass effects, they are rarely accompanied by external wounds.

Although pseudotumors can occur with any type of material or implant in total hip arthroplasty (THA), the majority of cases reported were caused by wear of the metal debris on the bearing surfaces and disassembled components of modular hip implants $[2,3]$. This article reports a rare case of pseudotumor formation with an ulcerative lesion caused by metal debris and fragments of a broken metal cable after revision THA. To the best of our knowledge, a cyst and synovial chondromatosis have been reported with a Dall-Miles cable grip system (Stryker Orthopaedics, Mahwah, NJ, USA), a multifilament cable used to reattach the osteotomized greater trochanter $[4,5]$, but pseudotumor formation has never been reported. We describe our experience in dealing with large pseudotumors caused by breakage of the Dall-Miles cable after revision THA. The study was performed in accordance with the principles of the Declaration of Helsinki. Informed consent for use and publication of photographs was obtained from the patient.

\section{Case Report}

Received: August 2, 2021

Revised: August 16, 2021

Accepted: August 16, 2021

Corresponding author:

Seong Oh Park, M.D., Ph.D.

Department of Plastic and Reconstructive Surgery, Hanyang University College of Medicine, 222-1 Wangsimni-ro, Seongdong-gu, Seoul 04763, Korea Tel: +82-2-2290-8567

Fax: +82-2-2290-8464

E-mail: psopark950@gmail.com

This is an Open Access article distributed under the terms of the Creative Commons Attribution Non-Commercial License (https://creativecommons org/licenses/by-nc/4 O/) which permits unrestricted non-commercial use, distribution, and reproduction in any medium, provided the original work is properly cited.

C) 2021 Korean Wound Management Society 


\section{Case}

\section{Patient information}

A 47-year-old female patient was referred to the clinic with complaints of pain and palpable swelling with an ulcerative lesion on the left thigh that began 2 months prior to presentation. The patient had undergone both metal-on-metal THA 24 years ago because of severe hip joint pain and limited motion caused by rheumatoid arthritis. Later, revision ceramic-on-ceramic THA with trochanteric fixation using a Dall-Miles cable was performed because of loosening of the implants 9 years prior. The patient had been on methotrexate and steroid therapy for rheumatoid arthritis since her diagnosis 30 years ago.

\section{Clinical findings}

Upon initial clinical examination, a $3 \times 3 \mathrm{~cm}$-sized suppurative ulcer and a palpable mass were found on the anteromedial aspect of the patient's left thigh (Fig. 1). Physical examination revealed no other complaints or instability of the hip joint. The patient did not complain of chills, fever, or systemic symptoms. Initial laboratory findings showed that the white blood cell (WBC) count was within normal limits, but some inflammatory markers were elevated; WBC count was $8.4 \times 10^{3} / \mathrm{mm}^{3}$ (normal range, $4-10 \times 10^{3} / \mathrm{mm}^{3}$ ), erythrocyte sedimentation rate (ESR) was $72 \mathrm{~mm} / \mathrm{hr}$ (normal, $<20 \mathrm{~mm} / \mathrm{hr}$ ), and C-reactive protein (CRP) level was $1.39 \mathrm{mg} / \mathrm{dL}$ (normal, $<0.5 \mathrm{mg}$ / dL). Methicillin-resistant Staphylococcus epidermidis was cultured from the external wound.

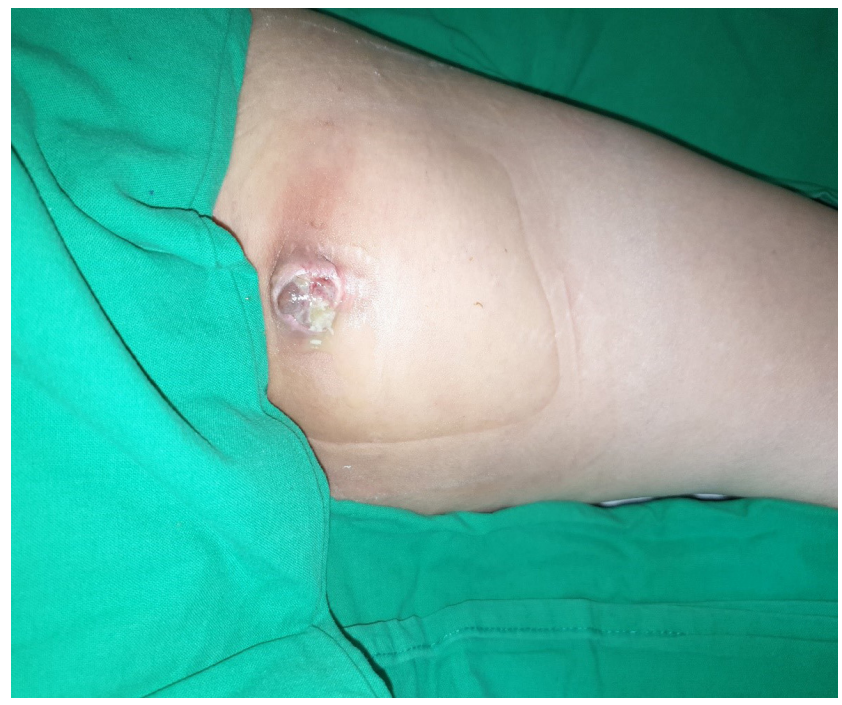

Fig. 1. Initial findings. A $3 \times 3 \mathrm{~cm}$-sized suppurative ulcerative lesion on the medial surface of the left thigh.

\section{Timeline}

\section{Diagnostic assessment}

An ultrasound scan of the left thigh confirmed a heteroechoic encapsulated cystic mass with internal debris (Fig. 2A). Radiography of the thigh showed breakage of the Dall-Miles cable grip system that had been used to fix the trochanteric osteotomy. Metal debris and multiple filaments of the broken cable were disseminated and had migrated to the medial aspect of the left thigh (Fig. 2B). Computed tomography (CT) demonstrated a $5.8 \times 14.2 \times 12.1 \mathrm{~cm}$-sized mass medial to the left thigh. The irregularly-shaped mass contained deposits of met-
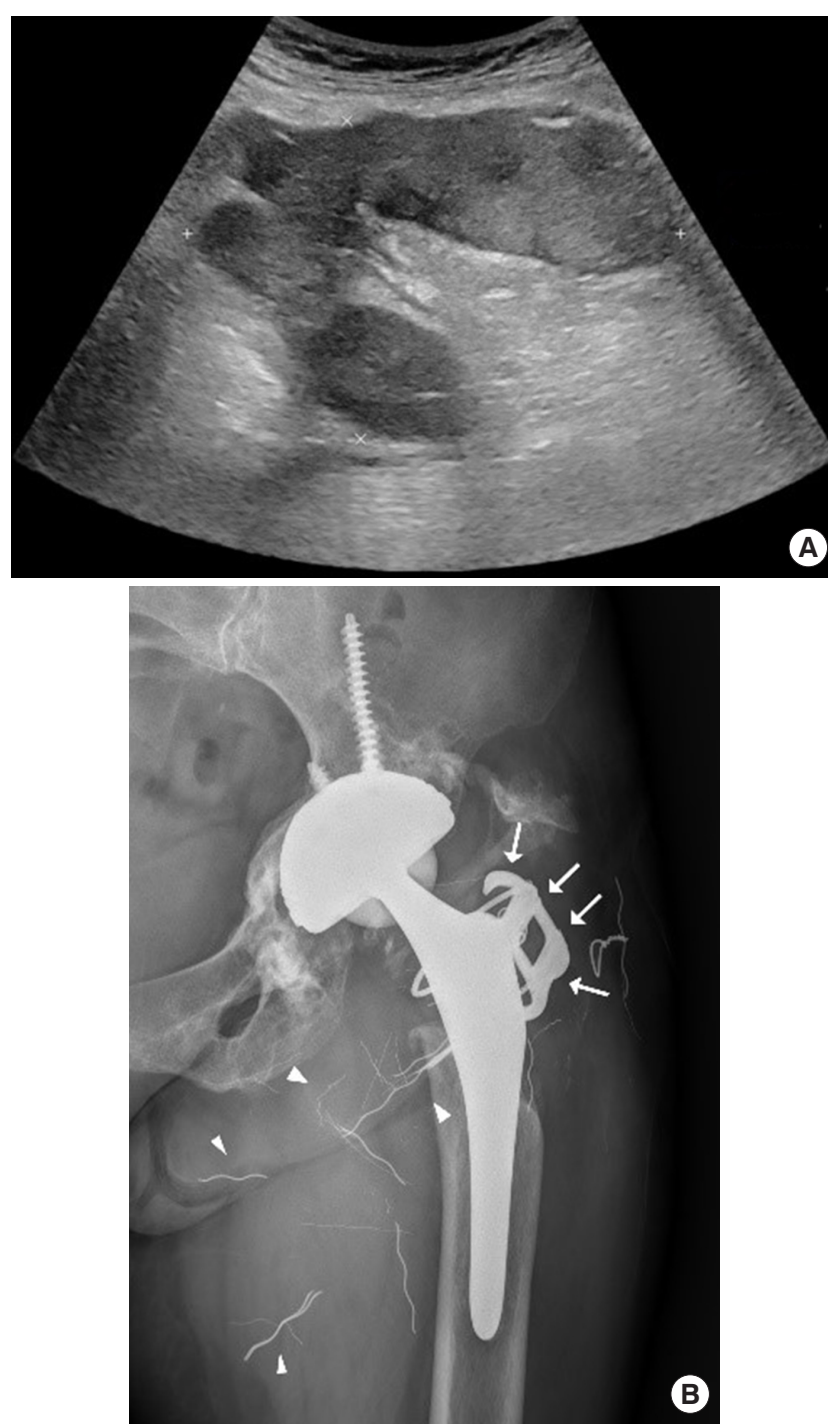

Fig. 2. Preoperative findings. (A) An ultrasound scan shows an irregularly-shaped cystic mass with posterior acoustic enhancement. (B) Anteroposterior radiography of the left thigh shows the Dall-Miles cable grip system (arrows) and multiple metal fragments of the broken cable (arrowheads). 
al debris and multiple metal filaments. Furthermore, the mass appeared to communicate with the ulcerative lesion and had invaded into the adductor longus muscle (Fig. 3A). Enhanced CT showed low-density central tissue and peripheral enhancement. There was no lymphatic enlargement or inflammatory signs in the surrounding tissues (Fig. 3B).

\section{Therapeutic intervention}

We performed surgical resection of the mass, including the external ulcerative wound. The extent of the mass ranged from an ulcerative lesion to the deep parts of the adductor longus muscle. The mass involved more than $70 \%$ of the adductor longus muscle, and the dissection was performed to completely remove the mass while preserving the nearby neurovascular structures. However, a portion of the mass extended deep to the posterior aspect of the femur and could not be approached. A specimen measuring $4 \times 11.5 \times 7 \mathrm{~cm}$ was removed, but a small remnant mass remained. After resection, the skin and large fascial defects were reconstructed using a fasciocutaneous advancement flap.

Histopathological examination of the resected specimen revealed granulomatous inflammation with metallic debris and lymphocytic infiltration, but there were no neoplastic features (Fig. 4). The mass was confirmed to be a pseudotumor originating from the deposition of metal debris.

After postoperative administration of intravenous antibiot- ics, the ESR and CRP levels improved to within the normal range, and the pain subsided. The wound healed completely and the patient was discharged without any other complications.

\section{Follow-up and outcomes}

Nine months after the first operation, during outpatient follow-up, the patient complained of recurrent pain with a palpable mass in the left thigh. The patient had no fever, and the laboratory findings revealed a WBC count within the normal range, with a mildly elevated ESR (64 mm/hr) and CRP level (1.59 mg/dL). CT scans revealed a recurrent pseudotumor with internal metal fragments in the adductor muscle in the left thigh (Fig. 5). We performed a second operation to approach the recurrent pseudotumor from the anterior and posterior sides, and completely resected it deep in the adductor muscle and removed the surrounding metal fragments (Fig. 6). After the second operation, the pain in the left thigh subsided and the surgical wound healed well without complications. Four months later, there was no evidence of recurrence of the pseudotumor on a CT scan (Fig. 7). Until the 8-month follow-up period, the patient did not complain of any symptoms or clinical problems. Furthermore, no other complications have risen due to the THA implant. The motion of the hip joint was within the normal range, and the patient has no problems walking to date.
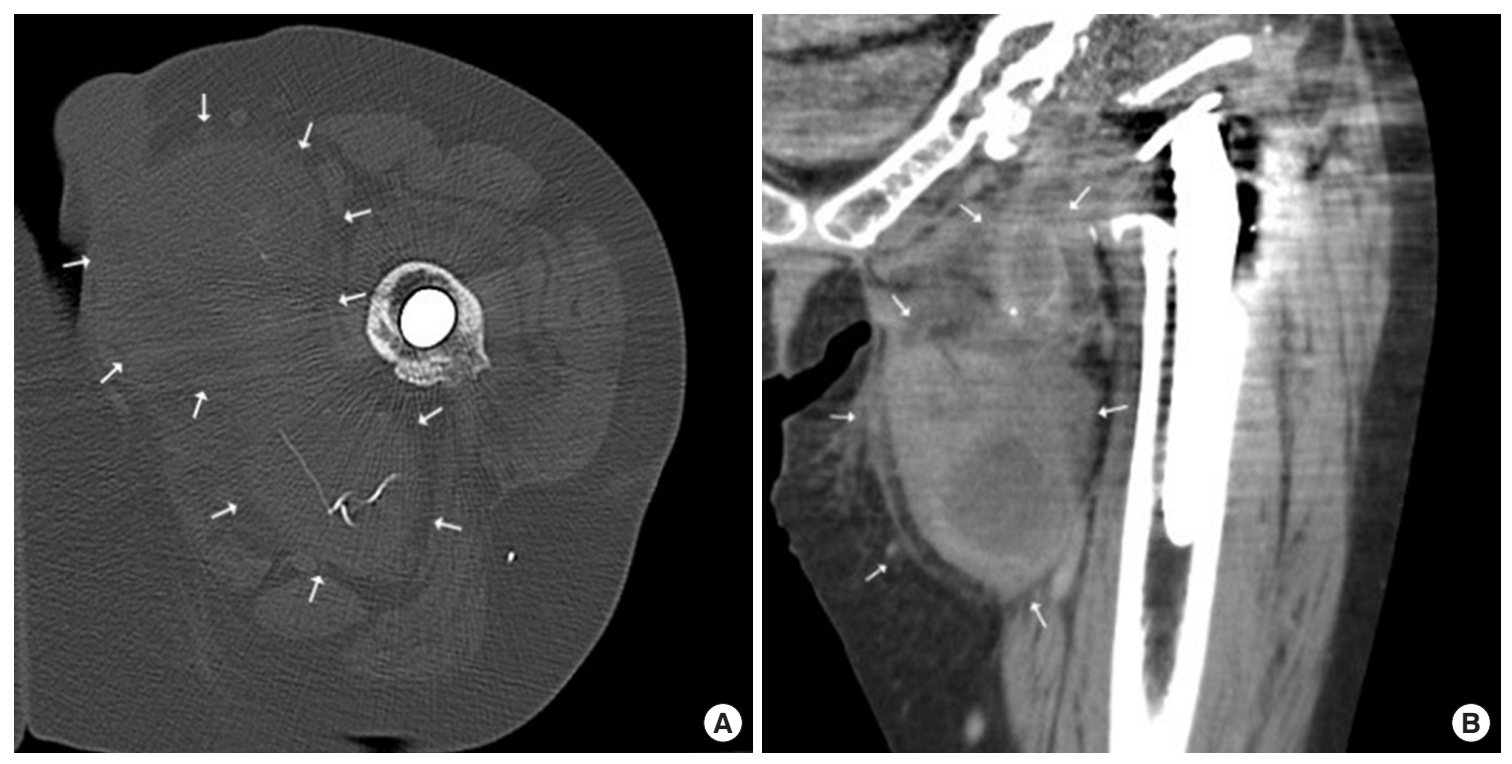

Fig. 3. Preoperative computed tomography (CT) of left hip. (A) A transverse view of non-contrast CT shows a $5.8 \times 14.2 \times 12.1 \mathrm{~cm}-$ sized mass with internal metal fragments (arrows). (B) A coronal view of enhanced CT shows invasion of the peripheral enhanced large mass into the adductor longus muscles (arrows). 
Oh SW et al.

Pseudotumor in Dall-Miles cable breakage
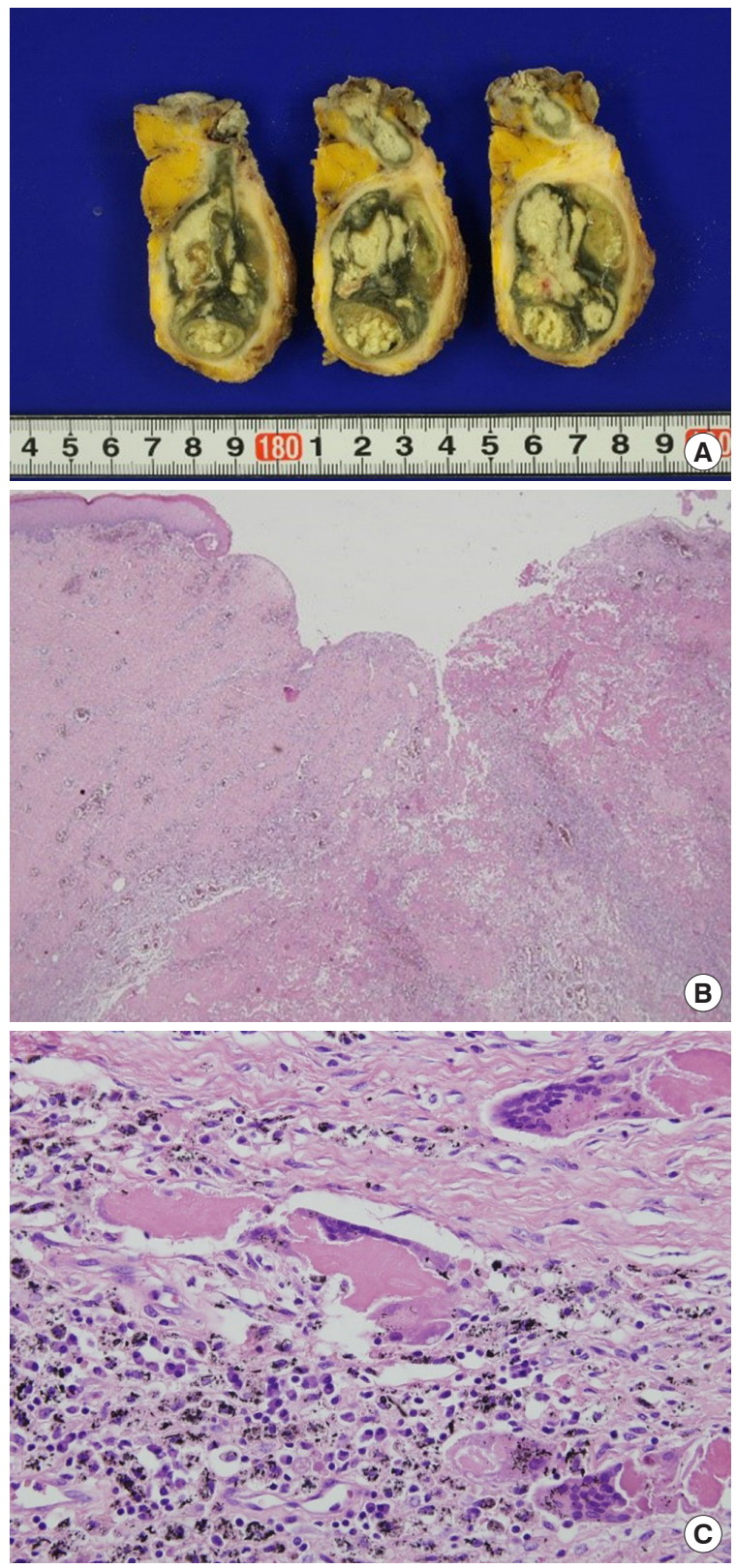

Fig. 4. Biopsy findings. (A) Gross specimens show a granuloma with black pigments and multifocal necrosis. (B) Acute and chronic inflammation with ulceration $(H \& E, \times 40)$. (C) Widespread lymphoplasmacytic and histiocytic infiltration with metallic black pigments and foreign-body reaction (H\&E, ×400).

\section{Discussion}

Pseudotumors can be caused by any type of material or component of orthopedic implants [6]. This rare case describes a large pseudotumor that was caused by breakage of the DallMiles cable grip system after revision THA. Revision THA requires reattachment of the trochanteric osteotomy using a
JWMR

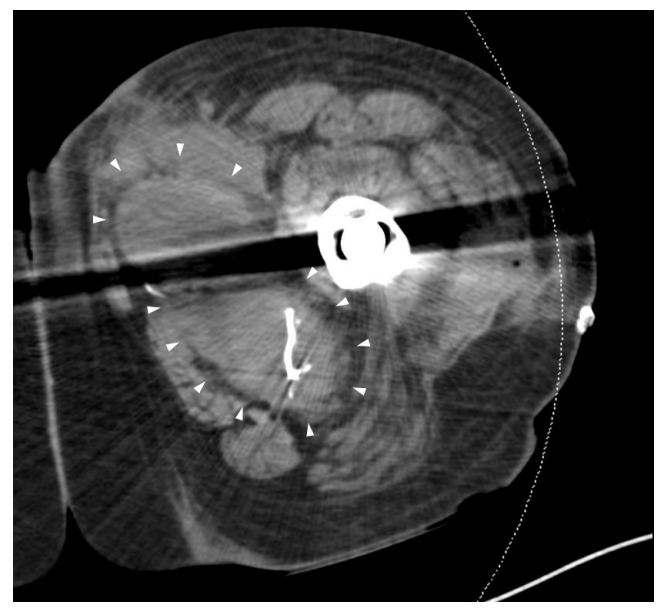

Fig. 5. Computed tomography of recurred pseudotumor. Recurrent pseudotumor with internal metal fragments on the posteromedial aspect of the left thigh (arrowheads).

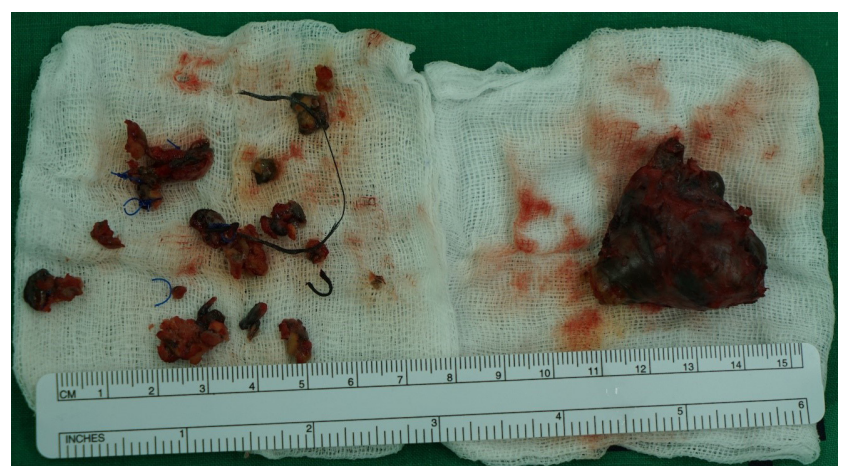

Fig. 6. Specimens from the secondary operation. Removed metal filaments and fragments of the broken Dall-Miles cable and the recurred pseudotumor.

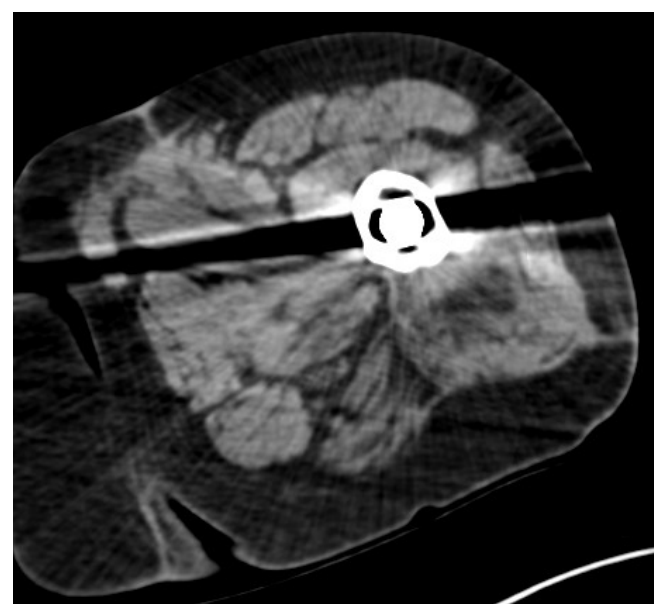

Fig. 7. Postoperative computed tomography (CT) of the left hip. Four months after the second operation, there is no evidence of recurrence of pseudotumor, and complete removal of metal fragment is confirmed through a CT scan. 
Dall-Miles cable [7]. In this case, multiple fragments of the broken Dall-Miles cable were disseminated in the periprosthetic soft tissue. Wear and corrosion of these metal fragments occurred, and the deposition of metal debris led to the pseudotumor.

Foreign-body reaction and type-IV hypersensitivity to metal and polyethylene debris are important in the pathogenesis of pseudotumors [1]. Keegan et al. [8] suggested that metals or polyethylene wear debris can be generated by mechanical wear and surface corrosion of orthopedic materials, and this is the primary cause of granulomatous reactions with histopathologic changes. Macrophages phagocytose excessive debris leading to the formation of granuloma, and the released prostaglandin E2 leads to periprosthetic osteolysis and implant loosening. The histological features of such pseudotumors include macrophage giant cells with inclusion of metal particles and diffuse perivascular infiltration of lymphocytes and plasma cells [1].

The incidence and prevalence of pseudotumors are unknown. A recent study reported that pseudotumors were found in $1 \%-5 \%$ of patients who undergo metal-on-metal hip resurfacing $[6,9]$. Common clinical symptoms include discomfort and a palpable swelling around the hip or the groin. A progressive, large pseudotumor may place pressure on neurovascular structures, resulting in neuropathy or venous thrombosis and dislocation or disassembly of the implant [10]. In the present case, the patient complained of pain and a palpable mass, but had no symptoms related to neuropathy or pressure effects. Furthermore, it is noteworthy that the pseudotumor was accompanied by an ulcerative lesion. Coexisting external wounds are rarely found. However, in this case, the metal fragment of the broken cable had spread and migrated into the superficial layer; hence, the pseudotumor involved to the layers of the skin. A differential diagnosis of a soft-tissue infection was made in this case due to the ulcer.

The pseudotumor should be differentiated from neoplasm and soft-tissue problems such as an abscess, tendinitis, and bursitis. The radiographic appearance of the pseudotumor may be helpful in that regard. Relevant radiographic features include the "bubble sign," a line caused by the deposition of metal debris [11]. In CT, a dense outline of metal debris or internal metal fragments can be identified clearly. In this case, the bubble sign was not clearly confirmed in the radiograph, but metal debris and multiple fragments of broken cable were identified. Pseudotumor formation with internal metal filaments was confirmed using CT.
The treatment of pseudotumors varies depending on the patient's symptoms and implant status. A small asymptomatic pseudotumor can be left for further observation, but if the size is large enough to compress neurovascular structures or if its symptoms are severe, complete removal of the pseudotumor and debridement of material debris and fragments are required to prevent recurrence. If corrosion is severe and loosening or joint instability occurs, revision joint arthroplasty using implants of other materials should also be considered.

In this case, despite the breakage of the Dall-Miles cable, the implant was intact without any instability or limited movement of the hip joint, so only pseudotumor resection was performed without THA revision. In the first operation, due to insufficient surgical exposure, the deep-seated pseudotumor in the posterior aspect of the femur was not completely resected and multiple filaments of a broken cable could not be sufficiently removed. Nine months later, the pseudotumor recurred. In the second operation, a sufficient surgical field was secured through simultaneous anterior and posterior approaches, complete resection was performed, and the metal filaments were removed completely.

This case described a pseudotumor caused by broken DallMiles cable that exhibited the possibility of pseudotumor formation on any type of orthopedic material and implant component. It also shows that pseudotumors may be accompanied by ulcerative lesions. If the patient complains of pain and has a palpable mass around an orthopedic implant, a diagnosis of a pseudotumor should be considered along with other differential diagnoses. In addition, it was confirmed that incomplete resection of pseudotumors and inadequate removal of orthopedic materials can contribute to pseudotumor recurrence.

\section{Conflict of interest}

No potential conflict of interest relevant to this article was reported.

\section{ORCID iDs}

Se Won Oh

Seung Yun Jee

https://orcid.org/0000-0002-5358-085X

Seong Oh Park

https://orcid.org/0000-0003-3895-817X

https://orcid.org/0000-0001-8990-0635

\section{References}

1. Daniel J, Holland J, Quigley L, et al. Pseudotumors associ- 
ated with total hip arthroplasty. J Bone Joint Surg Am 2012; 94:86-93.

2. Desai BR, Sumarriva GE, Chimento GF. Pseudotumor recurrence in a post-revision total hip arthroplasty with stem neck modularity: a case report. World J Orthop 2020;11: 116-22.

3. Eltit F, Wang Q, Wang R. Mechanisms of adverse local tissue reactions to hip implants. Front Bioeng Biotechnol 2019;7:176.

4. Wilson J, Riff AJ, Hellman MD, et al. A novel complication of the Dall-Miles cable grip system mimicking recurrent synovial chondromatosis: a case report and review of the literature. JBJS Case Connect 2016;6:e87.

5. Koyama K, Higuchi F, Kubo M, et al. Reattachment of the greater trochanter using the Dall-Miles cable grip system in revision hip arthroplasty. J Orthop Sci 2001;6:22-7.

6. Pandit H, Glyn-Jones S, McLardy-Smith P, et al. Pseudotumours associated with metal-on-metal hip resurfacings. J Bone Joint Surg Br 2008;90:847-51.
7. Kim IS, Pansey N, Kansay RK, et al. Greater trochanteric reattachment using the third-generation cable plate system in revision total hip arthroplasty. J Arthroplasty 2017;32: 1965-9.

8. Keegan GM, Learmonth ID, Case CP. Orthopaedic metals and their potential toxicity in the arthroplasty patient: a review of current knowledge and future strategies. J Bone Joint Surg Br 2007;89:567-73.

9. Kwon YM, Ostlere SJ, McLardy-Smith P, et al. "Asymptomatic" pseudotumors after metal-on-metal hip resurfacing arthroplasty: prevalence and metal ion study. J Arthroplasty 2011;26:511-8.

10. Counsell A, Heasley R, Arumilli B, et al. A groin mass caused by metal particle debris after hip resurfacing. Acta Orthop Belg 2008;74:870-4.

11. Heffernan EJ, Alkubaidan FO, Nielsen TO, et al. The imaging appearances of metallosis. Skeletal Radiol 2008;37:5962. 